\title{
OPTIC FLOW DOMINATES VISUAL SCENE POLARITY IN CAUSING ADAPTIVE MODIFICATION OF LOCOMOTOR TRAJECTORY
}

\author{
Nomura $\mathrm{Y}^{1}$, Mulavara $\mathrm{AP}^{2}$, Richards $\mathrm{JT}^{3}$, Brady $\mathrm{R}^{3}$, Bloomberg $\mathrm{JJ}^{4}$ \\ ${ }^{1}$ Nihon University School of Medicine, Tokyo, Japan $173-8610,{ }^{2}$ National Space \\ Biomedical Research Institute, Houston, TX, USA; ${ }^{3}$ Wyle Life Sciences, Inc., Houston, \\ TX 77058, USA; ${ }^{4}$ NASA Johnson Space Center, Houston, TX 77058, USA;
}




\begin{abstract}
Locomotion and posture are influenced and controlled by vestibular, visual and somatosensory information. Optic flow and scene polarity are two characteristics of a visual scene that have been identified as being critical in how they affect perceived body orientation and self-motion. The goal of this study was to determine the role of optic flow and visual scene polarity on adaptive modification in locomotor trajectory. Two computer-generated virtual reality scenes were shown to subjects during 20 minutes of treadmill walking. One scene was a highly polarized scene while the other was composed of objects displayed in a non-polarized fashion. Both virtual scenes depicted constant rate self-motion equivalent to walking counterclockwise around the perimeter of a room. Subjects performed Stepping Tests blindfolded before and after scene exposure to assess adaptive changes in locomotor trajectory. Subjects showed a significant difference in heading direction, between pre and post adaptation stepping tests, when exposed to either scene during treadmill walking. However, there was no significant difference in the subjects' heading direction between the two visual scene polarity conditions. Therefore, it was inferred from these data that optic flow has a greater role than visual polarity in influencing adaptive locomotor function.
\end{abstract}




\section{Introduction}

Locomotion and posture are influenced and controlled by vestibular, visual and somatosensory information $[18,23,24,27,38,48]$. Visual input information consists of various factors including variation in type and distribution of the content of the scene. Several characteristics of patterned visual scenes have been identified as being critical in how they affect perceived body orientation and self-motion [23]. These factors perceived from the visual scene are then integrated in the central nervous system and provide input for postural and locomotor control. Visual scene characteristics can be distinguished into two kinds of categories; those that contribute to optic flow and those that define polarity of the visual scene.

\section{The role of optic flow in postural and locomotor control}

Locomotion results from the output synergy of multiple sensory inputs, including vision. Visual inputs provide us with important cues for orientation and self-movement perception during locomotion. The patterned visual motion seen during self-movement constitutes the optic flow field that provides perceptual cues about self-movement and environmental structure [17].

Artificially imposed optic flow can cause changes in perceived body orientation and the sense of self movement (vection), when it conflicts with information from other sensory inputs. Studies have shown that subjects who are sitting and are stationary experience illusory self-tilt when viewing a real rotating circular display of dots $[20,22]$. Astronauts have reported increased sensations of body-tilt and self-rotation when viewing a rotating display of dots in microgravity. This is attributed to an increased reliance on 
vision resulting from the lack of gravity input that otherwise provides a reference for equilibrium information $[32,33,47,50]$.

Other studies have directly measured the effects of optic flow on postural stability during locomotion. The rate at which people walk on a self-driven treadmill has been shown to depend on the velocity of an artificial optic flow pattern along the line of sight relative to their walking speed [35]. Linear optic flow has also been shown to cause directionally specific postural sway and positional deviations during treadmill locomotion $[4,5,44,45]$. The effects of rotational optic flow have also been demonstrated. When oscillated in roll and viewed during quiet stance, scenes containing complex, realistic content caused more postural sway than scenes with simple radial patterns [14]. While walking over ground in a stereoscopic virtual environment that rotated in roll, subjects showed compensatory torso rotation in the direction of scene rotation that resulted in positional variation away from a desired linear path [26].

\section{The role of visual scene polarity in postural and locomotor control}

An object is said to have visual polarity, or to be "visually polarized", when it contains an identifiable principal axis with one end distinct from the other [23]. Most visually polarized objects, such as houses, tables, and chairs appear symmetrical in the vertical plane and have distinct ends that are designated "top" and "bottom". Usually, we are familiar with the visual scene distribution being organized according to certain rules including the assumption that the ceiling of a room is located upward, the floor is located downward and the furniture is placed on the floor. This polarized scene distribution is never tilted unless we lean our head or body with respect to the gravity force axis. In this 
context, the vestibular, proprioceptive and tactile sensors informs the central nervous system that the body is indeed not vertical. However, the perception of subjective vertical and postural equilibrium can be influenced if the visual scene is distorted or displays tilted polarity [34]. For instance, entering a room consisting of an oblique wall, ceiling and floor would lead to the perception of standing obliquely [13]. Similar to pure optic flow, visual polarity cues can have compelling effects on an observer's perceived spatial orientation. If all polarized objects in view are statically tilted with respect to gravity by the same amount, static observers sitting upright with respect to the gravity vector feel as if they themselves are tilted [3].

There is also evidence that visual polarity can strengthen the sense of self-motion one perceives while viewing a dynamic visual scene. Subjects who sat in a real room that constantly rotated around them reported more rotational self-motion when that room was furnished with polarized objects than when it did not contain such objects [24]. Duh et al. reported that their subjects exhibited more balance disturbance and more difficulty in maintaining posture during viewing a rotating high resolution virtual reality scene containing polarized objects than when they viewed a simple radial pattern [14]. We have previously reported difference in the influence of a polarized furnished room scene and a random dots non-polarized scene moving about its different axes with polarized scenes causing greater postural instability than the non-polarized scenes during treadmill walking [36]. 


\section{Adaptive modification of locomotor trajectory}

Humans can rapidly readjust and recalibrate various characteristics of gross movement activities such as walking to deal with variations in environmental constraints by using visual input $[15,18,44,45]$. Some of these characteristics include the estimation of distances to walk to reach a target and the direction of movement $[37,38]$.

Rieser et al demonstrated that the estimate of distance to a target, to which the subject has to walk, can be recalibrated by exposure to a disparity between visual flow and walking speed [38]. Subjects walked on a motorized treadmill and were exposed to linear visual flow that moved either faster or slower than their biomechanical walking speed. After 8 minutes of exposure to this stimulus subjects were asked to walk in a straight line to a remembered target location with vision occluded. Subjects exposed to visual flow that was faster than their treadmill walking speed tended to overshoot the remembered target while the converse was true for those subjects exposed to visual flow slower than their walking speed. This experiment showed that an adaptive recalibration occurred in subject's perception of forward translation after exposure to brief period of adaptive visual stimuli.

Additionally, other studies have shown adaptive recalibration in locomotor trajectory. Subjects that were asked to step in place on a rotating disk with their head and torso aligned with their straight ahead direction inadvertently turned in circles when asked to step in place on a stationary surface with eyes closed after exposure to this stimulus. This is an adaptive effect called podokinetic after-rotation (PKAR) $[18,48]$. PKAR suggests a remodeling of the somatosensory signals regarding the relationship between trunk rotation with respect to the feet and the perception of trunk rotation 
relative to space [48]. Other investigators have reported changes in walking trajectory following exposure to even simple visual stimuli consisting of optokinetic black and white vertical stripe patterns when the subjects were seated [19,25]. After exposure to this optokinetic stimulus subjects showed deviated stepping in the direction opposite to the previously viewed visual scene movement as measured by a controlled Fukuda step test [16]. These results demonstrate that exposure to even simple optokinetic stimuli when seated can induce adaptive deviation of locomotor trajectory as an after effect.

In a recent study we examined if a plastic adaptive modification in locomotor performance could be achieved by viewing a virtual environment scene that rotated about the subjects yaw axis of rotation during treadmill locomotion [30]. Two groups of subjects walked on a motorized treadmill for 20 minutes while viewing the interior of a $3 \mathrm{D}$ virtual scene projected onto a screen in front of them. The virtual scene depicted constant self-motion equivalent to either: 1) walking around the perimeter of a room to one's left (ANGULAR) or, 2) walking down the center of a hallway (LINEAR). The forward tangential velocity of the rotating room and the forward velocity of the virtual hallway were equivalent. During the treadmill locomotion adaptation period, each subject's torso yaw angular rotation was measured. For both groups, five step trials were completed before and after the treadmill locomotion adaptation period. During step trials, subjects marched in place to the beat of a metronome at 90 steps per minute while blindfolded in a quiet room. The subject's final heading direction was measured for each trial. During post treadmill stepping task, subjects in the ANGULAR group as compared to the LINEAR group: 1) showed significantly greater deviation during post exposure testing in the heading direction and $\mathrm{Y}$ position opposite to the direction of optic 
flow experienced during treadmill walking and 2) showed a significant monotonically increasing torso yaw angular rotation bias in the direction of optic flow during the treadmill adaptation exposure period. Thus, viewing a rotating virtual environment during treadmill walking caused adaptive plastic modifications in the central interpretation of the heading direction relationship relative to the straight ahead as defined by the vestibular, proprioceptive and visual input inducing adaptive changes in locomotor trajectory.

These studies show that optic flow and visual polarization influence dynamic postural equilibrium during locomotion and the underlying adaptive processes that contribute to locomotor trajectory. However, it is still unknown how changes in optic flow and polarity influence adaptive changes in locomotor function. Therefore, the goal of this study was to determine the roles of optic flow and visual scene polarity on adaptive modification in locomotor trajectory. To answer this question we developed two kinds of computer generated virtual reality scenes: the Gravity Virtual Reality (GVR) scene and Non - Gravity Virtual Reality (NGVR) scene. The GVR was a fully furnished room with a well-defined ceiling and floor with familiar objects (desks, chairs, lamp, pictures etc.) - distributed as they would be in a normal office scene. The NGVR scene contained the same office objects displayed in a non-polarized fashion with the contents distributed randomly. Importantly, the two scenes contained the same objects, the only difference being their orientation with respect to gravity. 


\section{Materials and Methods}

\section{Subjects}

37 healthy subjects of average $( \pm 1 \mathrm{SD})$ age $=32.0( \pm 5.9)$ yr. with normal or corrected-to-normal vision were recruited from the Human Test Subject Facility at Johnson Space Center (JSC) in Houston, Texas. Subjects were randomly assigned to two groups. One group watched the Gravity Virtual Reality (GVR, $n=20)$ scene and the other group watched the Non-Gravity Virtual Reality (NGVR, $n=17)$ scene while they walked on a treadmill. The experimental protocol was approved by the JSC Committee for the Protection of Human Subjects (CPHS), and informed consent was obtained prior to testing.

\section{Treadmill Adaptation Protocol}

Subjects walked on a motorized treadmill (Gaitway/Kistler, Amherst, NY) with a surface area of $50 \times 152 \mathrm{~cm}$. They walked at $4.0 \mathrm{~km} / \mathrm{h}$ while viewing either the GVR or NGVR visual scene. The scenes remained static for the first 4 minutes to allow the subjects to get used to walking on the treadmill. The scene then rotated at a constant rate of $30 \mathrm{deg} / \mathrm{s}$ for the remaining 20 minutes continuously. Subjects were instructed to watch the scene for the entire trial and try to immerse themselves in it without paying attention to their position on the treadmill belt. A spotter provided guiding commands to make sure the subject stayed at the approximate center of the treadmill when they approached the limits of the treadmill belt surface. To prevent injury through falling, each subject wore a full-body harness that was attached to an overhead gantry that provided support and stopped the treadmill if the subject fell. During usual performance this harness did not 
interfere with the natural movement of the body and walking. The treadmill had a handrail on its left edge, but subjects were instructed not to touch it unless it was completely necessary. The subjects wore head-mounted sport goggles with the plastic lens removed to occlude the view of peripheral objects and to help maximize immersion in the virtual scenes.

\section{Virtual Reality Scene Generation}

The GVR scene was a room richly furnished in visual content, with realistic texture-mapped objects such as desks, chairs, trees, and gravity-cued pictures with a tile pattern floor (Fig. 1A). The second scene (NGVR) was the same size and shape, containing exactly the same furniture, potted plants, pictures, floor pattern and ceiling as the GVR. The sole difference between the two scenes was that the contents of the NGVR room were distributed in space and oriented randomly with respect to the gravity vector as if the room existed in a non-gravity environment (Fig 1B).

The monoscopic, passive-immersive 3D Virtual Reality Scenes were created using graphic modeling software (3ds max 4; Discreet, Montreal, Que.) and rendered using virtual environment software (Vizard v.2, Python v. 2.0) on a PC computer (2.2 GHz, Intel Pentium 4 processor, nVIDIA Quadro2 EX graphics card). The GVR and NGVR scenes were back-projected onto a screen positioned $1.5 \mathrm{~m}$ in front of the subject's eyepoint using a digital DP 1100 projector (Kodak, Inc., Rochester, NY). Those projected scenes were $65^{\circ} \mathrm{H} \mathrm{x} 48^{\circ} \mathrm{V}$ field-of-view (FOV) and were viewed by subjects on the treadmill. Scenes constantly rotated in yaw as if the subjects wcre walking in a room along a curved wall in the counterclockwise direction. Both the GVR and NGVR 
virtual rotating rooms were cylindrically shaped with a simulated diameter of $20 \mathrm{~m}$ and height of $4 \mathrm{~m}$ and a rotating speed of 30 degrees per second to match with the treadmill walking speed. Previous studies have shown rotation rates close to this to be effective in causing sensations of self-motion $[30,36]$. The subject's simulated eyepoint was displaced $1.8 \mathrm{~m}$ above, $7 \mathrm{~m}$ left and $1 \mathrm{~m}$ back from the center of the both of the room's floor as it rotated about an axis coincident with its center, creating the illusion that the subject was walking around the perimeter of the room to his or her left.

\section{Stepping Test Protocol}

Subjects completed five Stepping Tests before and after exposure to the Treadmill Adaptation Protocol. While wearing blindfolds and ears-plugs, subjects were instructed to march in place to the sound of a metronome which was attached to their back and beating at 90 steps per minute for a total of 100 steps per trial. At the end of each trial, the subject's final position and the heading direction of their feet were marked. After that, the subject, still blindfolded, was walked back to the origin by the test operator who led them in a random pattern to prevent feedback of performance. The subject was then placed back on the origin such that they were facing the same direction at the beginning of each trial with feet parallel and toes pointing forward.

Upon completing the Treadmill Adaptation Protocol, subjects were immediately blindfolded and wore ear plugs, and moved in a chair with wheels to the area where the Stepping Test was conducted in order to preserve the adaptive effects produced during treadmill walking. After finishing the Stepping Test, the subject's final position (Xfore/aft, Y- left/right) relative to the initial position $(0 \mathrm{~cm}, 0 \mathrm{~cm})$ and the intercepts of the 
line $\left(\mathrm{X}_{\mathrm{int}}, \mathrm{Y}_{\mathrm{int}}\right)$ indicating the heading orientation direction with respect to the initial heading orientation direction (straight ahead $\tilde{=} 0 \mathrm{deg}$ ) were measured (see Fig. 2).

\section{Data Analysis}

The recorded final positions $(X, Y)$ and the intercepts of the line $\left(X_{\text {int }}, Y_{\text {int }}\right)$ indicating the heading orientation direction with the global axes, were used to calculate the heading orientation direction angle (HD) for each trial. The HD was calculated and coded for the final direction of the Stepping Test as follows:

$$
\begin{aligned}
& \Theta_{c W}=\Theta^{*}-\frac{\Pi}{2}, X>0 \\
& \Theta_{c W}=\Theta^{*}-\frac{3 \Pi}{2}, X<0 \\
& \Theta_{c c W}=\Theta^{*}+\frac{3 \Pi}{2}, X>0 \\
& \Theta_{c c W}=\Theta^{*}+\frac{\Pi}{2}, X<0 \\
& \Theta^{*}=a \tan \left(\frac{Y-Y_{\text {int }}}{X-X_{\text {int }}}\right)
\end{aligned}
$$

$\mathrm{CW}$ and $\mathrm{CCW}$ indicate the clockwise and counter clockwise directions of the subjects' movement with respect to their initial positions. These data obtained during the Stepping Tests were analyzed using ANOVA (Within subject factors - Adaptation, 2 levels: pre and post; vs Across subject factor - Group: two levels - GVR and NGVR) in order to determine the influence of visual scene polarity on adaptive locomotor function. A student $t$ - test was performed on the difference between the pre and post adaptation measures for the three variables. Data were analyzed at a significance level of .05 using a standard statistical software package (SPSS v. 10.0, Chicago, IL). 


\section{Results}

Figure 3 shows the average ( \pm one standard error of the mean) of the final heading direction (HD, deg: 3A), final mediolateral position $(X, \mathrm{~cm}: 3 \mathrm{~B})$ and the final fore-aft position $(\mathrm{Y}, \mathrm{cm}: 3 \mathrm{C})$ across all subjects pre and post exposure to the visual scene adaptation protocol for the two groups (GVR scene and NGVR scene). Repeated measures ANOVA on HD revealed there was a significant main effect of ADAPTATION for $\operatorname{HD}\left[\mathrm{F}_{(1,35)}=42.591, \mathrm{p}<0.0005\right]$. However, there was no significant interaction for Group x Adaptation for HD ( $\mathrm{p}>0.05)$. A student $\mathrm{t}$ - test on the difference between the pre and post adaptation HD revealed a significant difference for both groups $(\mathrm{p}<0.05)$.

Repeated measures ANOVA on $\mathrm{X}$ position revealed there was a significant main effect of ADAPTATION for $\mathrm{X}$ position $\left[\mathrm{F}_{(1,35)}=20.852, \mathrm{p}<0.0005\right]$. However, there was no significant interaction for Group x Adaptation for X position $(\mathrm{p}>0.05)$. A student $\mathrm{t}$ - test on the difference between the pre and post $\mathrm{X}$ position revealed a significant difference for both groups $(\mathrm{p}<0.05)$.

Repeated measures ANOVA on Y position revealed there was a significant main effect of ADAPTATION $\left[\mathrm{F}_{(1,35)}=4.858, \mathrm{p}<0.034\right]$. However, there was no significant interaction for Group X Adaptation for Y position $(\mathrm{p}>0.05)$. A student $t$ - test on the difference between the pre and post $\mathrm{Y}$ position revealed a significant difference only for the NGVR group $(\mathrm{p}<0.05)$ but remained not significant for the GVR group $(\mathrm{p}>0.05)$.

\section{Discussion}

The goal of this study was to determine the role of optic flow and visual scene polarity on adaptive modification in locomotor trajectory. Two computer-generated 
virtual reality scenes: the GVR and NGVR scenes were shown to subjects during 20 minutes of treadmill walking. The GVR was a highly polarized scene while the NGVR scene was composed of objects displayed in a non-polarized fashion; the two scenes contained the same objects, the only difference being their apparent polarized distribution. Subjects performed Stepping Tests without the aid of vision pre and post scene exposure to assess adaptive changes in locomotor trajectory.

Subjects showed a significant difference in heading direction, between pre and post adaptation Stepping Tests, when exposed to both GVR and NGVR scenes during treadmill walking. On the other hand, there was no significant difference in the subjects' heading direction between GVR and NGVR conditions. Therefore, it was inferred from these data that optic flow has a greater role than visual polarity in influencing adaptive locomotor function.

\section{The effects of visual scene polarity in controlling locomotor trajectory}

Viewing a rotating polarized scene influences postural sway to a greater extent than a non- polarized scene while standing and walking [14,36]. However, these previous studies did not factor in the contents of the visual scenes. Whenever a rotating visual scene is viewed, optokinetic nystagmus are generated to counter the optic flow to help stabilize vision. Previous studies on eye movement control have shown that optokinetic nystagmus caused by optic flow could be varied by the visual scene's contents including variation in color, luminance, density, and distribution of the scene contents in the center or peripheral retinal locations $[29,31,42,43,46]$. These variations in scenc content may similarly induce differential effects in adaptive locomotor responses. Therefore, in the 
current study both the polarized and non- polarized scenes were designed to contain the same objects, considering optokinetic stimulation as a factor that induces variations in optokinetic nystagmus.

Despite these controls in this study, no significant difference in adaptation was found to be caused by differences in polarity. Our previous study had shown a significant difference in the strategic torso stabilization responses during locomotion control when viewing polarized scenes in comparison to viewing non-polarized scenes that were rotated about or translated along all the degrees of freedom [36]. Other studies have shown the importance of optic flow and that optic flow in a virtual scene, clearly influences locomotion trajectory [44]. These studies compared patterns of optic flow in virtual scenes presented to subjects via a head mounted display and analyzed how these influence locomotor trajectories while subjects were viewing scenes. They found that subjects steered their walking trajectory towards the intended target and showed that additional visual flow had a greater role in the control of steering of locomotor trajectory. Recent studies have also suggested that the perception of tilt and that of self rotation may be processed independently by visual-vestibular subsystems [41]. The results from our study support this hypothesis as differences in polarity of objects in the optic flow pattern rotating about the subjects vertical axis resulted in no differential adaptive effect. We hypothesize that the presence of other veridical information with respect to the gravity vector while walking on the treadmill may have contributed to the lack of differences. In support of this observation, astronauts' responses of illusory self motion and the simultaneous compensation for these motions when viewing a rotating display of dots on the inside of rotating drum, or vertical optokinetic stimulation gave way to responses that 
are reported in 1-G, when braces or bungee were used to replicate the missing axial ground pressure forces [49].

\section{The role of optic flow in controlling locomotor trajectory}

In this study, subjects showed a deviation in the stepping directions after exposure to both GVR and NGVR yaw direction rotating adaptive stimuli while walking on a linear treadmill. Despite stepping at 90 steps/min during the post treadmill Step Tests, subjects remained unaware of the leftward deviation and forward movement and actually believed that they had been stepping in place throughout the stepping tests. However, exposure to optic flow in the static seated position also causes alteration of locomotor trajectory in the after effect period with similar unawareness of subjects during controlled Fukuda stepping test $[19,25]$. These studies have reported adaptive changes in step rotation angle with the direction of the deviation tendency counter to the visual stimuli direction. Their results indicate that optic flow is sufficient to induce an adaptive deviation in locomotor trajectory as after-effect, in the direction counter to the optokinetic stimuli. Several studies investigating the after-effects of jogging, including those using the podokinetic adaptive paradigm with and without concurrent optokinetic stimulation have reported similar results $[1,15,18,24,48]$. Other studies from our laboratory reported that during exposure to a polarized rotating visual optic flow, similar to that used in the current study, during walking on a linear treadmill induced a torso bias in the direction of optic stimulation [30]. Subjects, from this study, also showed a significant deviation in locomotor trajectory as well as forward translation when performing stepping tests after the exposure period. 
A number of studies have shown the transfer of adaptation between oculomotor stabilization systems and transfer from these systems to other motor control responses. There are numerous examples of optokinetic stimulus causing modification of gain of the vestibulo-ocular reflex [40] and modification of the VOR affecting the gain of the optokinetic nystagmus response [2]. Also, previous research on a visuomotor task of pointing has shown a transfer of adaptation to the manual control system after modifying the gain of the VOR [6]. These studies demonstrate the potential for adaptive transfer between various motor systems and suggest a common neural site for the processing and self-motion perception and concurrent adaptation in motor output.

Various cortical areas have been implicated in the processing of this synergetic interplay between optic flow and other sensory modalities. The dorsal stream of primate cortex contains motion processing areas that are selective for optic flow and self-motion [28]. One such functionally specialized area is the ventral intraparietal area (VIP) within the posterior parietal cortex of the primate [10]. The VIP contains many neurons that show directionally selective discharges to stimuli from multiple modalities including moving visual, tactile, vestibular, or auditory stimuli. Functional imaging data on humans also reveal a network of cortical areas that respond to polymodal stimuli conveying motion information. One of these regions of activations is located in the depth of human intraparietal sulcus [10]. Accordingly, these authors have suggested that this area constitutes the human equivalent of primate area VIP. Studies of monkeys strongly suggested that VIP responds to optic flow and is involved in the encoding of self motion and heading direction $[7,8,39,51,52]$. In addition to VIP, the medial superior temporal area of extrastriate cortex (MST) is suggested to contain sufficient information for 
threshold judgments of optic flow [21], and is suggested to have a role in processing of heading direction and perception of self motion $[9,11,12]$. These cortical areas maybe involved in providing information for locomotor heading direction and may be involved in the adaptive transfer between motor output modalities. Therefore, vision, vestibular and somatosensory information may be processed as a weighted sum and therefore produce a perceived change in heading direction when the central interpretation of one of the sensory channels is modified. Importantly, this change in heading direction may not be perceived by other sensory modalities (i.e. vestibular) because of central reinterpretation of self-motion perception induced by exposure to optic flow. 


\section{Acknowledgements}

This study was supported in part by NASA and through NASA Cooperative Agreement NCC9-58 with the National Space Biomedical Research Institute by grants to Jacob J Bloomberg (PI). We also wish to thank the subjects for contributing their time participating in this study. 


\section{References}

[1] Anstis, S., Aftereffects from jogging, Exp Brain Res, 103 (1995) 476-8.

[2] Aoki, H. and Yagi, T., Effect of VOR gain changes on OKR gain control in human subjects, Adv Otorhinolaryngol, 41 (1988) 58-62.

[3] Asch, S.E. and Witkin, H.A., Studies in space orientation. II. Perception of the upright with displaced visual fields and with body tilted, J Exp Psychol Gen, 121 (1992) 407-18; discussion 404-6.

[4] Bardy, B.G., Warren, W.H., Jr. and Kay, B.A., Motion parallax is used to control postural sway during walking, Exp Brain Res, 111 (1996) 271-82.

[5] Bardy, B.G., Warren, W.H., Jr. and Kay, B.A., The role of central and peripheral vision in postural control during walking, Percept Psychophys, 61 (1999) 1356-68.

[6] Bloomberg, J.J., Merkle, L.A., Barry, S.R., Huebner, W.P., Cohen, H.S., Mueller, S.A. and Fordice, J., Effects of adaptation of vestibulo-ocular reflex function on manual target localization, J Vestib Res, 10 (2000) 75-86.

[7] Bremmer, F., Duhamel, J.R., Ben Hamed, S. and Graf, W., Heading encoding in the macaque ventral intraparietal area (VIP), Eur J Neurosci, 16 (2002) 1554-68.

[8] Bremmer, F., Klam, F., Duhamel, J.R., Ben Hamed, S. and Graf, W., Visual-vestibular interactive responses in the macaque ventral intraparietal area (VIP), Eur J Neurosci, 16 (2002) 1569-86.

[9] Bremmer, F., Kubischik, M., Pekel, M., Lappe, M. and Hoffmann, K.P., Linear vestibular self-motion signals in monkey medial superior temporal area, Ann N Y Acad Sci, 871 (1999) 272-81. 
[10] Bremmer, F., Schlack, A., Duhamel, J.R., Graf, W. and Fink, G.R., Space coding in primate posterior parietal cortex, Neuroimage, 14 (2001) S46-51.

[11] Britten, K.H. and van Wezel, R.J., Electrical microstimulation of cortical area MST biases heading perception in monkeys, Nat Neurosci, 1 (1998) 59-63.

[12] Britten, K.H. and Van Wezel, R.J., Area MST and heading perception in macaque monkeys, Cereb Cortex, 12 (2002) 692-701.

[13] Dichgans, J. and Brandt, T.H., Visual-Vestibular Interactions: Effects on Self-Motion Perception and Postural Control. In R. Held, H. Leibowitz and H. Teuber (Eds.), Handbook of Sensory Physiology, Vol. VIII, Springer Verlag, Berlin, 1978, pp. 755-804.

[14] Duh, H.B., Lin, J.J., Kenyon, R.V., Parker, D.E. and Furness, T.A., Effects of characteristics of image quality in an immersive environment, Presence Teleoper Virtual Environ, 11 (2002) 324-32.

[15] Durgin, F.H. and Pelah, A., Visuomotor adaptation without vision?, Exp Brain Res, 127 (1999) 12-8.

[16] Fukuda, T., The stepping test: two phases of the labyrinthine reflex, Acta Otolaryngol, 50 (1959) 95-108.

[17] Gibson, J., The senses considered as perceptual systems, Houghton Mifflin, Boston, 1966.

[18] Gordon, C.R., Fletcher, W.A., Melvill Jones, G. and Block, E.W., Adaptive plasticity in the control of locomotor trajectory, Exp Brain Res, 102 (1995) 540-5.

[19] Gordon, C.R., Tal, D., Gadoth, N. and Shupak, A., Prolonged optokinetic stimulation generates podokinetic after rotation, Ann N Y Acad Sci, 1004 (2003) 297-302.

[20] Held, R., Dichigans, J. and Bauer, J., Characteristics of moving visual scenes influencing spatial orientation, Vision Res, 15 (1975) 357-65. 
[21] Heuer, H.W. and Britten, K.H., Optic flow signals in extrastriate area MST: comparison of perceptual and neuronal sensitivity, J Neurophysiol, 91 (2004) 1314-26.

[22] Howard, I.P., Cheung, B. and Landolt, J., Influence of vection axis and body posture on visually induced self rotation, Advisory group for aerospace research and development, $433(1988) 1-15$.

[23] Howard, I.P. and Childerson, L., The contribution of motion, the visual frame, and visual polarity to sensations of body tilt, Perception, 23 (1994) 753-62.

[24] Jurgens, R., Boss, T. and Becker, W., Podokinetic after-rotation does not depend on sensory conflict, Exp Brain Res, 128 (1999) 563-7.

[25] Kato, I., Miyoshi, T. and Pfaltz, C.R., Studies on habituation of vestibulospinal reflexes. Effects of repetitive optokinetic and vestibular stimuli upon the stepping test, ORL $J$ Otorhinolaryngol Relat Spec, 39 (1977) 195-202.

[26] Keshner, E.A. and Kenyon, R.V., The influence of an immersive virtual environment on the segmental organization of postural stabilizing responses, J Vestib Res, 10 (2000) 20719.

[27] Lackner, J.R. and DiZio, P., Visual stimulation affects the perception of voluntary leg movements during walking, Perception, 17 (1988) 71-80.

[28] Lappe, M., Bremmer, F. and van den Berg, A.V., Perception of self-motion from visual flow, Trends Cogn Sci, 3 (1999) 329-336.

[29] Miyoshi, T., Pfaltz, C.R. and Piffko, P., Effect of repetitive optokinetic stimulation upon optokinetic and vestibular responses, Acta Otolaryngol, 75 (1973) 259-65. 
[30] Mulavara, A.P., Richards, J.T., Marshburn, A.N., Regina, B. and Bloomberg, J.J., Adaptive effects on locomotion performance following exposure to a rotating virtual environment. Annual meeting of American Society of Biomechanics, Toledo, 2003.

[31] Murasugi, C.M. and Howard, I.P., Up-down asymmetry in human vertical optokinetic nystagmus and afternystagmus: contributions of the central and peripheral retinae, Exp Brain Res, 77 (1989) 183-92.

[32] Oman, C.M., Howard, I.P., Carpenter-Smith, T., Beall, A.C., Natapoff, A., Zacher, J.E. and Jenkin, H.L., Neurolab experiments on the role of visual cues in microgravity spatial orientation. In J.C. Buckey and J.L. Homick (Eds.), The Neurolab Spacelab mission: Neuroscience research in space: results from the STS-90, Neurolab Space mission, Vol. NASA SP-2003-535, NASA Aeronautics and Space Administration, lyndon B. Johnson Space Center, Houston, 2003.

[33] Oman, C.M., Lichtenberg, B.K., Money, K.E. and McCoy, R.K., M.I.T./Canadian vestibular experiments on the Spacelab-1 mission: 4 . Space motion sickness: symptoms, stimuli, and predictability, Exp Brain Res, 64 (1986) 316-34.

[34] Parker, D.E. and Poston, R.L., Tilt from a head-inverted position produces displacement of visual subjective vertical in the opposite direction, Percept Psychophys, 36 (1984) $461-5$.

[35] Prokop, T., Schubert, M. and Berger, W., Visual influence on human locomotion. Modulation to changes in optic flow, Exp Brain Res, 114 (1997) 63-70.

[36] Richards, J.T., Mulavara, A.P. and Bloomberg, J.J., Postural stability during treadmill locomotion as a function of the visual polarity and rotation of a three-dimensional environment, Presence:Teleoperations and Virtual Reality, 13 (2004) 371-384. 
[37] Rieser, J.J., Ashmead, D.H., Talor, C.R. and Youngquist, G.A., Visual perception and the guidance of locomotion without vision to previously seen targets, Perception, 19 (1990) 675-89.

[38] Rieser, J.J., Pick, H.L., Jr., Ashmead, D.H. and Garing, A.E., Calibration of human locomotion and models of perceptual-motor organization, $J$ Exp Psychol Hum Percept Perform, 21 (1995) 480-97.

[39] Schlack, A., Hoffmann, K.P. and Bremmer, F., Interaction of linear vestibular and visual stimulation in the macaque ventral intraparietal area (VIP), Eur J Neurosci, 16 (2002) 1877-86.

[40] Schor, C. and Westall, C., Rapid adaptation of the vestibulo-ocular reflex and induced self-motion perception, Percept Psychophys, 40 (1986) 1-8.

[41] Thilo, K.V. and Gresty, M.A., Visual motion stimulation, but not visually induced perception of self-motion, biases the perceived direction of verticality, Brain Res Cogn Brain Res, 14 (2002) 258-63. .

[42] Wang, L., The relationship of luminous intensity and velocity for motion perception and maximum OKN elicitation, Vision Res, 31 (1991) 1601-9.

[43] Wang, L., Soderberg, P.G. and Tengroth, B., Influence of target direction, luminance and velocity on monocular horizontal optokinetic nystagmus, Acta Ophthalmol (Copenh), 71 (1993) 578-85.

[44] Warren, W.H., Jr., Kay, B.A., Zosh, W.D., Duchon, A.P. and Sahuc, S., Optic flow is used to control human walking, Nat Neurosci, 4 (2001) 213-6.

[45] Warren, W.H., Kay, B.A. and Yilmaz, E.H., Visual control of posture during walking: functional specificity, J Exp Psychol Hum Percept Perform, 22 (1996) 818-38. 
[46] Watanabe, Y., Ohmura, A., Shojaku, H. and Mizukoshi, K., Optokinetic nystagmus elicited by a random dot pattern and a wide interval stripe pattern in normal subjects, Acta Otolaryngol Suppl, 511 (1994) 104-8.

[47] Watt, D., Landolt, J. and Young, L., Effects of long term weightlessness on roll circularvection, Canadian Aeronautics Space Journal, 39 (1993) 52-55.

[48] Weber, K.D., Fletcher, W.A., Gordon, C.R., Melvill Jones, G. and Block, E.W., Motor learning in the "podokinetic" system and its role in spatial orientation during locomotion, Exp Brain Res, 120 (1998) 377-85.

[49] Young, L.R., Jackson, D.K., Groleau, N. and Modestino, S., Multisensory integration in microgravity, Ann N Y Acad Sci, 656 (1992) 340-53.

[50] Young, L.R., Oman, C.M., Watt, D.G., Money, K.E., Lichtenberg, B.K., Kenyon, R.V. and Arrott, A.P., M.I.T./Canadian vestibular experiments on the Spacelab-1 mission: 1. Sensory adaptation to weightlessness and readaptation to one-g: an overview, Exp Brain Res, 64 (1986) 291-8.

[51] Zhang, T. and Britten, K.H., Clustering of selectivity for optic flow in the ventral intraparietal area, Neuroreport, 15 (2004) 1941-5.

[52] Zhang, T., Heuer, H.W. and Britten, K.H., Parietal area VIP neuronal responses to heading stimuli are encoded in head-centered coordinates, Neuron, 42 (2004) 993-1001. 


\section{Legends}

Figure 1: The Gravitational Virtual Reality (GVR) scene (A) was a room richly furnished in visual content, with realistic texture-mapped objects such as desks, chairs, trees, and gravity-cued pictures with a tile pattern floor. Non- Gravitational Virtual Reality (NGVR) Scene (B) was the same size and shape containing exactly the same furniture, potted plants, pictures, floor pattern and ceiling as the GVR. The sole difference between the two scenes was that the contents of the NGVR room were distributed randomly in space as if the room existed in a non-gravity environment.

Figure 2: Axes orientation and measures calculated during the Stepping Test protocol. The subject's final position (Y - fore/aft, $\mathrm{X}$ - left/right) relative to initial position $(0 \mathrm{~cm}, 0$ $\mathrm{cm}$ ) and the heading direction with respect to the initial heading orientation direction (straight ahead $\tilde{=} 0 \mathrm{deg}$ ) were measured. Two examples are shown with $\mathrm{X}_{1}>0, \mathrm{Y}_{1}>0$, $\Theta_{1}<0, \mathrm{X}_{2}<0, \mathrm{Y}_{2}<0$, and $\Theta_{2}>0$.

Figure 3: The average ( \pm one standard error of the mean) of the final heading direction (HD, deg; A), final mediolateral (X, cm; B) position and the final fore-aft position (Y, $\mathrm{cm}$; C) across all subjects pre and post exposure to the visual scene adaptation protocol for the GVR group (closed squares, $\mathbf{a}$ ) and the NGVR group (closed diamond, $\bullet$ ). 
Figure

(A)

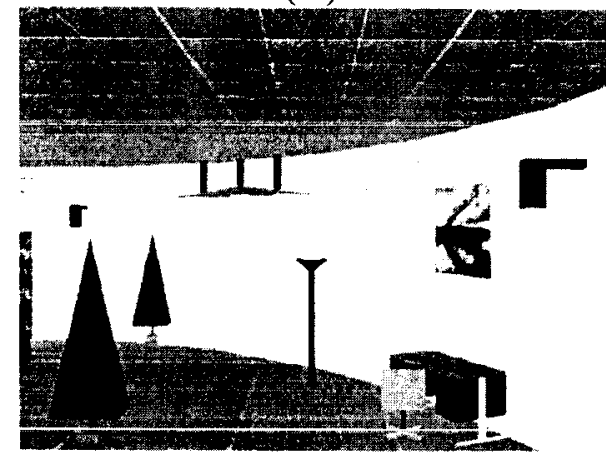

(B)

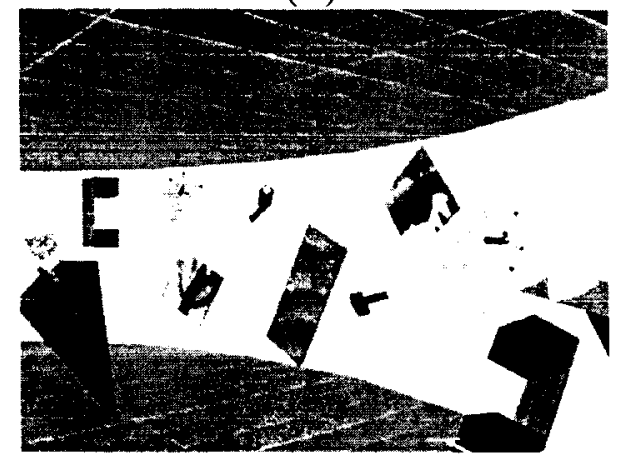

Figure 1 


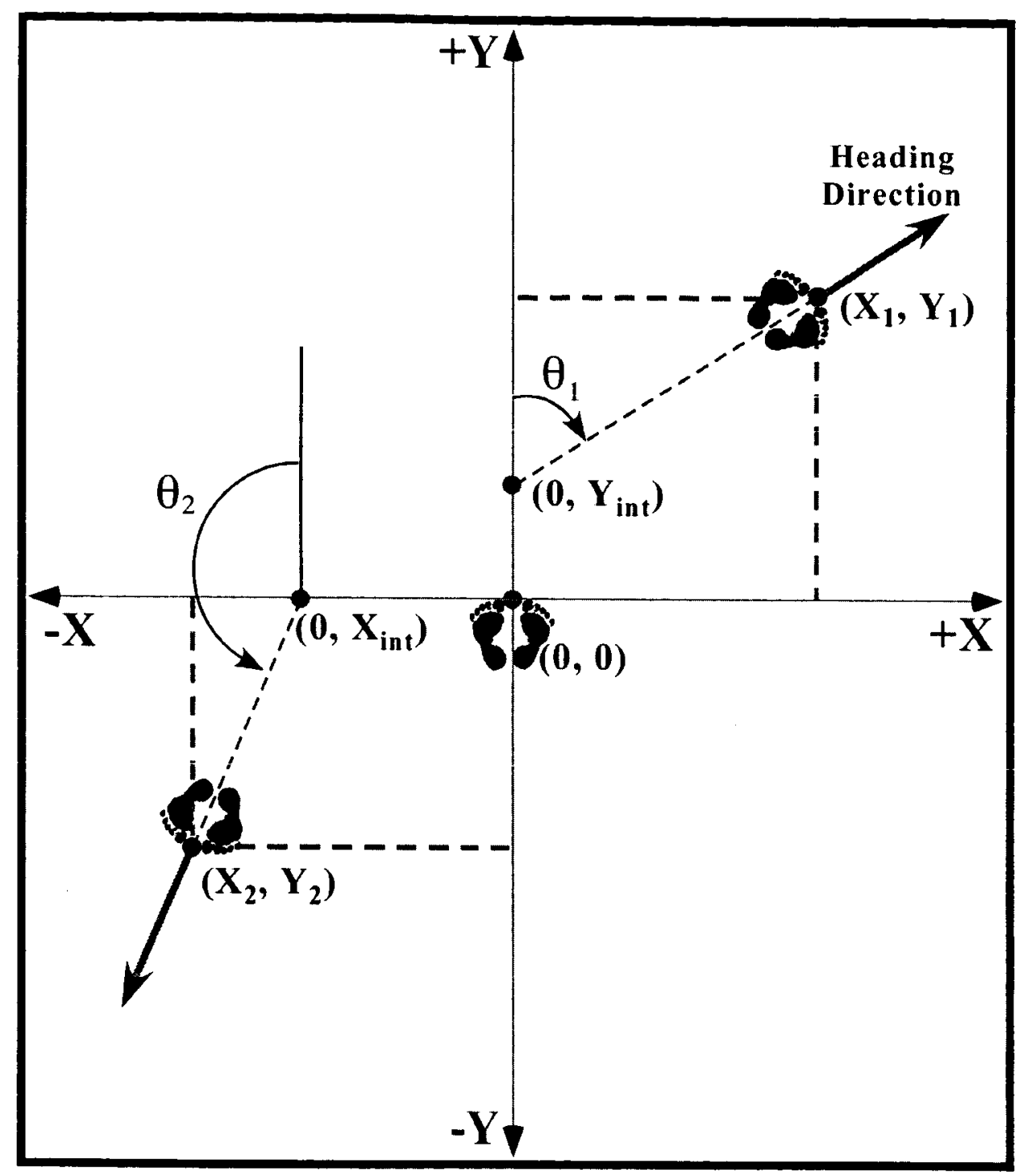

Figure 2 


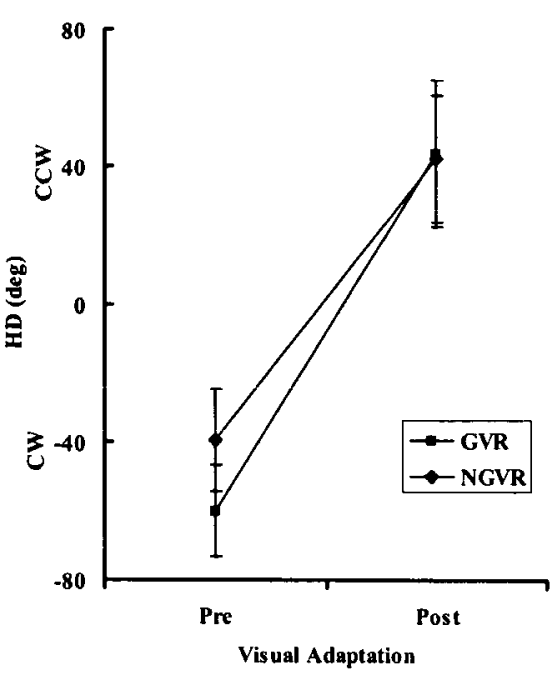

B

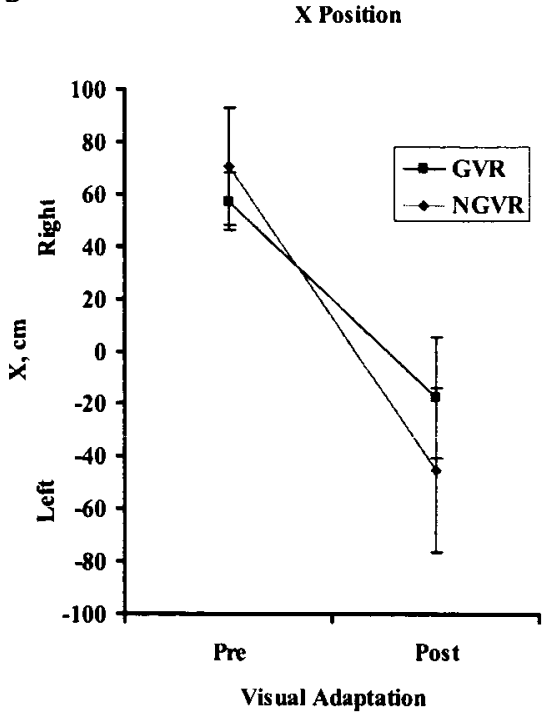

C

Y Position

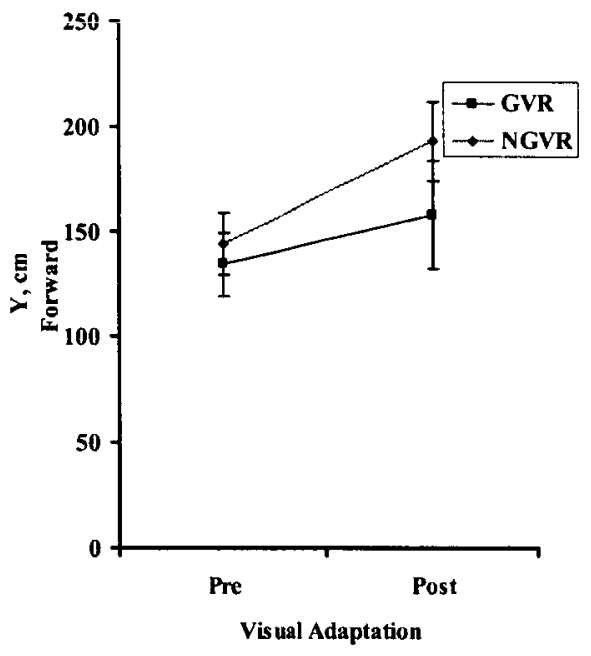

Figure 3 